\title{
Edible biofilm formation from guava seed waste fermentation
}

\author{
Sari Darmasiwi, Oktaviana Herawati, and Endah Retnaningrum* \\ Laboratory of Microbiology, Faculty of Biology, Universitas Gadjah Mada, 55281, \\ Yogyakarta, Indonesia \\ *e-mail: endahr@ugm.ac.id
}

\begin{abstract}
Guava seed is by-product from the consumption of guava fruits. We interested to explore further the potential of guava seed waste using fermentation method. The purpose of this research was to determine the ability of biofilm formation produced from fermentation of guava seed. Fermented guava seed was prepared by solid-state fermentation method using banana leaves wrap at $37{ }^{\circ} \mathrm{C}$ for $72 \mathrm{~h}$. It was then continued with isolation and screening of bacteria from the fermentation products, preparation of bacteria cultures to be used in biofilm formation, and formation of biofilm by glass slides and broth cultures methods. The edible biofilm formation by glass slide method was observed by light microscopy using $0.5 \%$ Crystal Violet dye, while biofilm formation by broth cultures method was observed by transmission electron microscopy (TEM) using phosphotungstic acid 2 $\%$ dye. The results show that there were 3 (three) strains lactic acid bacteria (LAB) candidates isolated from fermented guava seed waste product (J6, J7, and J8 strains). The observation by light microscopy showed that J7 strain was the only strain which was unable to form biofilm by glass slide method. All the strains showed the ability to form biofilms in different stages by broth cultures method. Thus, guava seed fermentation was able to produce edible biofilm but the LAB strains still need to be identified further.
\end{abstract}

\section{Keywords}

edible, biofilm, fermentation, guava seed, TEM

\section{Introduction}

Biofilm become scientist' interest due to their significance in nature. It has function as barriers covering some solid surfaces to protect

Citation: Darmasiwi, S., Herawati, 0., Retnaningrum, E.: Edible biofilm formation from guava seed waste fermentation. In: I.W. Mustika, I. Kartini. (eds.): Proceeding of the $3 \mathrm{rd}$ International Conference on Science and Technology, Vol. 1, pp. 39-43. UGM Digital Press Physical Science and Engineering, Yogyakarta (2018).

Published: October, 2018 microbial community from the environmental stresses. Biofilm can be developed in any surfaces such as plastic, metals, glasses, woods, soils, tissues and food materials, depends on the nutrient availability on the surfaces. To form biofilm, microorganisms should make attachment into the surfaces, mediated by pili, fimbriae, or flagella [1]. This aggregation of microorganisms is then releasing extra polymeric substances (EPS) matrix such as polysaccharides, lipids, proteins or combination from all of the substances which comprise about $85 \%$ volume, that create flexible layers over the surfaces. The compositions of the substances are correlated with species of microbes, age of biofilm formation and environmental factors [2].

Microbial biofilms are sometimes known to be responsible for health infections, deterioration of water quality and food contamination [1]. 
However, the positive roles of biofilm formation is not much known. Biofilm can be used in food and medicine as environmentally friendly and biodegradable materials. Biofilm can protect food from water, gas, lipid oxidation and odor [3]. The positive applications of biofilm depend on the strength and flexibility of biofilm, permeability against water vapor, and biofilm solubility in the water [4]. Thus, edible biofilm can be developed as alternatives for food preservation to prolong the shelf-time of fruit, vegetables and improve food quality [5].

Edible biofilm can be developed from indigenous microbes which refers to generally recognize as save (GRAS) microorganisms isolated from fruits, vegetables or another food materials. Guava (Psidium guajava) contains $12 \%$ of inedible seeds that usually consider as waste. According to [6], guava seeds may contain protein (9.73\% dry weight), glutelin globulin, albumin, and fibers [7]. Thus, there are still plenty nutrients contained in guava seeds. However, study on microbial diversity in guava seed, as well as its roles and application has not been done so far. Fermentation of seed may increase nutrients composition and lowering food toxicity. Thus, in this research we tried to isolate indigenous microorganisms from guava seed waste and explore the potential of guava seed waste by using fermentation to produce edible biofilm.

\section{Methods}

\subsection{Fermentation of Guava Seed Waste}

Guava seed waste was collected from local juice seller in Yogyakarta, Indonesia. Guava seeds were rinsed using tap water and air-dried overnight. After the seeds dried, they were grinded until become fine powder (flour). Fermentation were done by adding guava seed flour with sterile water (2:1), and subsequently the mixture was wrapped with banana leaves. Fermentation was carried out triplicate at $37{ }^{\circ} \mathrm{C}$ for $72 \mathrm{~h}$. Control was done triplicate using unfermented guava seed powder.

\subsection{Microbial Isolation and Screening from Guava Seed Waste Fermentation}

About $10 \mathrm{~g}$ of fermented guava seed were transferred aseptically and homogenated in $90 \mathrm{ml}$ sterile saline solution (5 g pepton, $8.5 \mathrm{~g} \mathrm{NaCl}$ and $1 \mathrm{~L}$ destilled water, $\mathrm{pH} \mathrm{7,0).} \mathrm{It} \mathrm{was} \mathrm{then} \mathrm{inoculated} \mathrm{aseptically} \mathrm{in}$ MRSAgar (Man Rogosa Sharpe Agar), incubated at $30{ }^{\circ} \mathrm{C}$ for 24 to $48 \mathrm{~h}$ followed by screening of microorganisms recognized as GRAS.

\subsection{Edible Biofilm Formation}

Edible biofilm formation was done on glass slides and in the broth culture. In order to made biofilm on glass slides, inoculum was transferred to sterile MRS Broth in petridish containing glass slides, incubated at 30 ${ }^{\circ} \mathrm{C}$ for $5 \mathrm{~d}$. After cultivation, glass slides were washed 3 (three) times using aquadest and dried at room temperature. To perform biofilm in broth culture were done by inoculation of potential isolates in MRS Broth in $1.5 \mathrm{~mL}$ microtube, incubated at $37^{\circ} \mathrm{C}$ for $5 \mathrm{~d}$.

\subsection{Observation of Biofilm Formation}

Dried biofilms on glass slides were observed by light microscopy, referred from the method of [8] with modification. To observed this, glass slides were stained using Crystal Violet $0.5 \%$ for 30 min, washed for 5 (five) times using aquadest, dried in room temperature, and observed by light microscopy. To preview biofilm in the broth culture, it was stained by phosphotungstic acid $2 \%$ dye and observed by transmission electron microscopy. 


\section{Results and Discussions}

\subsection{Microbial Screening}

Edible biofilm can be produced from probiotics or generally recognize as safe (GRAS) microorganisms so it is safe to be consumed. To do this, we tried to isolate and select probiotic/ lactic acid bacteria (LAB). Several genera included in LABs are Lactococcus, Enterococcus, Oenococcus, Pediococcus, Streptococcus, and Lactobacillus [9]. These bacteria can be grown by culturing in $\mathrm{MRSAgar}$ with $\mathrm{CaCO}_{3}$ addition. $\mathrm{CaCO}_{3}$ has function as indicator for lactic acid production from the microorganisms. $\mathrm{CaCO} 3$ will be dissolved by acid production resulting clear zone surrounding the microbial colonies. Preliminary screening showed that there were 8 (eight) strain candidates indicated as lactic acid bacteria (Table 1).

Table 1 Preliminary screening of LAB isolated from guava seed fermentation

\begin{tabular}{ccc}
\hline $\begin{array}{c}\text { Dilution } \\
\text { Factor }\end{array}$ & Strain & $\begin{array}{c}\text { Clear } \\
\text { zone } \\
\text { diameter }\end{array}$ \\
\hline $10^{-3}$ & $\mathrm{~J}-1$ & 0,10 \\
& $\mathrm{~J}-2$ & 0,10 \\
& $\mathrm{~J}-3$ & 0,05 \\
& $\mathrm{~J}-4$ & 0,10 \\
& $\mathrm{~J}-5$ & 0,05 \\
$10^{-4}$ & $\mathrm{~J}-6$ & 0,19 \\
& $\mathrm{~J}-7$ & 0,20 \\
& $\mathrm{~J}-8$ & 0,20 \\
\hline
\end{tabular}

J-6, J-7 and J-8 strains were the biggest strains that resulted clear zone diameters in MRS Agar. Those strains were then used as potential strains to produce edible biofilm. However further identification such as catalase tests and Gram staining should be done to confirm the strains as lactic acid bacteria.

\subsection{Biofilm Formation on Glass Slides}

Formation of biofilm on glass slides observed using light microscopy and Crystal Violet $0.5 \%$ were showed in Figure 1. It revealed that in J-6 and J-8 strains, biofilms were seen in purple color, since it was bounded to Crystal Violet $0.5 \%$ stain. The difference between J-6 strain was that biofilm in J-6 strain had formed micro-colony, a grape-like- compact structure with thick biofilm layers, while in J-8 strain, bacteria were still visible as individual and had not made any cluster that was the beginning step of biofilm formation. Moreover, in J-7 strain, purple color was not visible so that it did not show any biofilms production from the strain (Fig. 1). It showed that biofilm formation produced by microorganisms were strain-dependent. There were potential strains, or less potential in producing biofilm. 


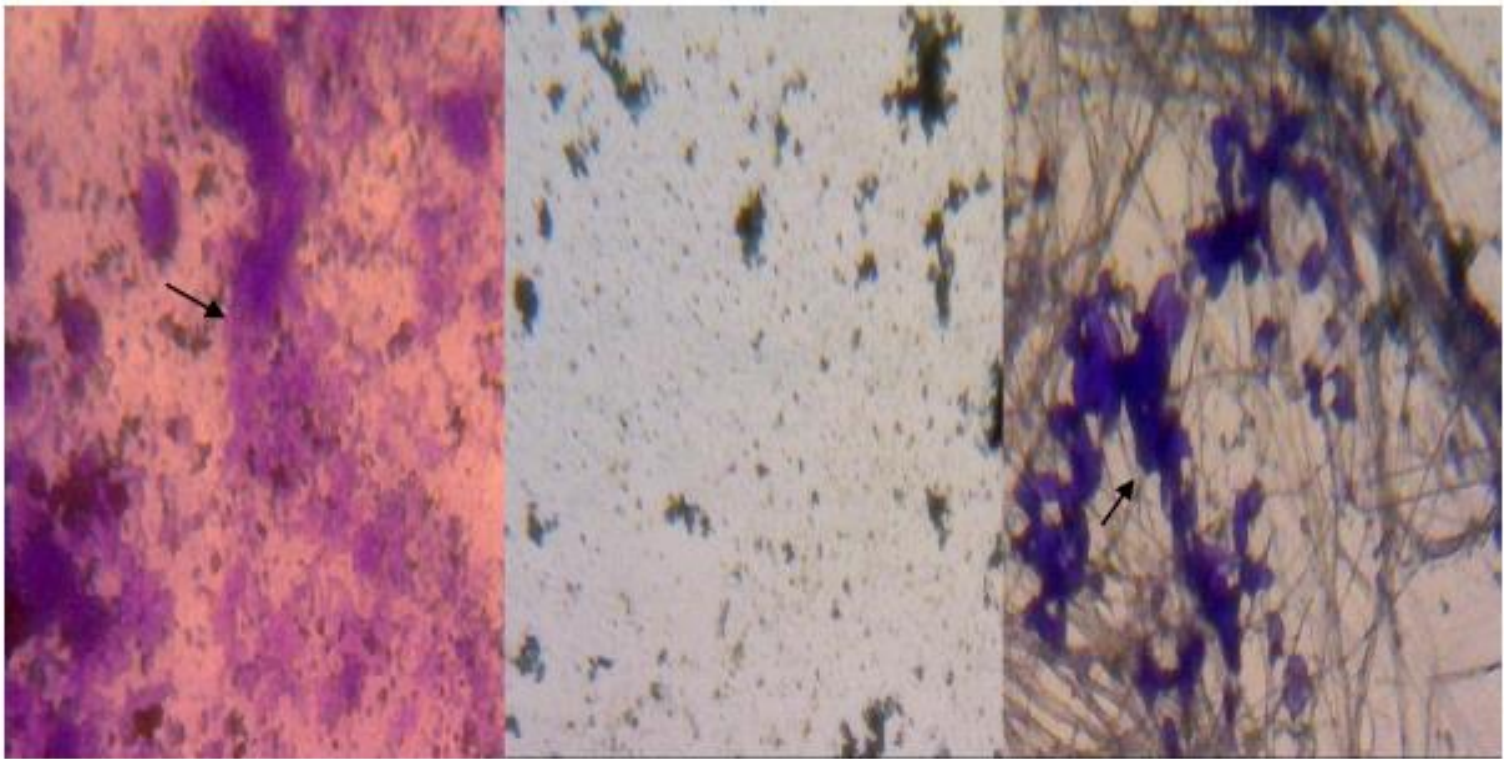

(A)

(B)

(C)

Fig. 1 Biofilm formation on glass slides observed with light microscopy and Cristal violet 0,5\%. a. J-6 strain showed clustered cells with thick biofilm layers, b. J-7 strain showed independent cells and did not form aggregation, c. J-8 strain showed cells aggregation, first phase of biofilm formation. Arrows showed biofilm structure

\subsection{Biofilm Formation in Broth Cultures}

In order to view biofilm in the broth cultures, potential strains J-6, J-7 and J-8 were inoculated in MRS Broth medium at $37^{\circ} \mathrm{C}$ for $5 \mathrm{~d}$. Observation by transmission microscopy electron showed that all the strains were capable to produce biofilm in different phase and forms (Fig. 2).

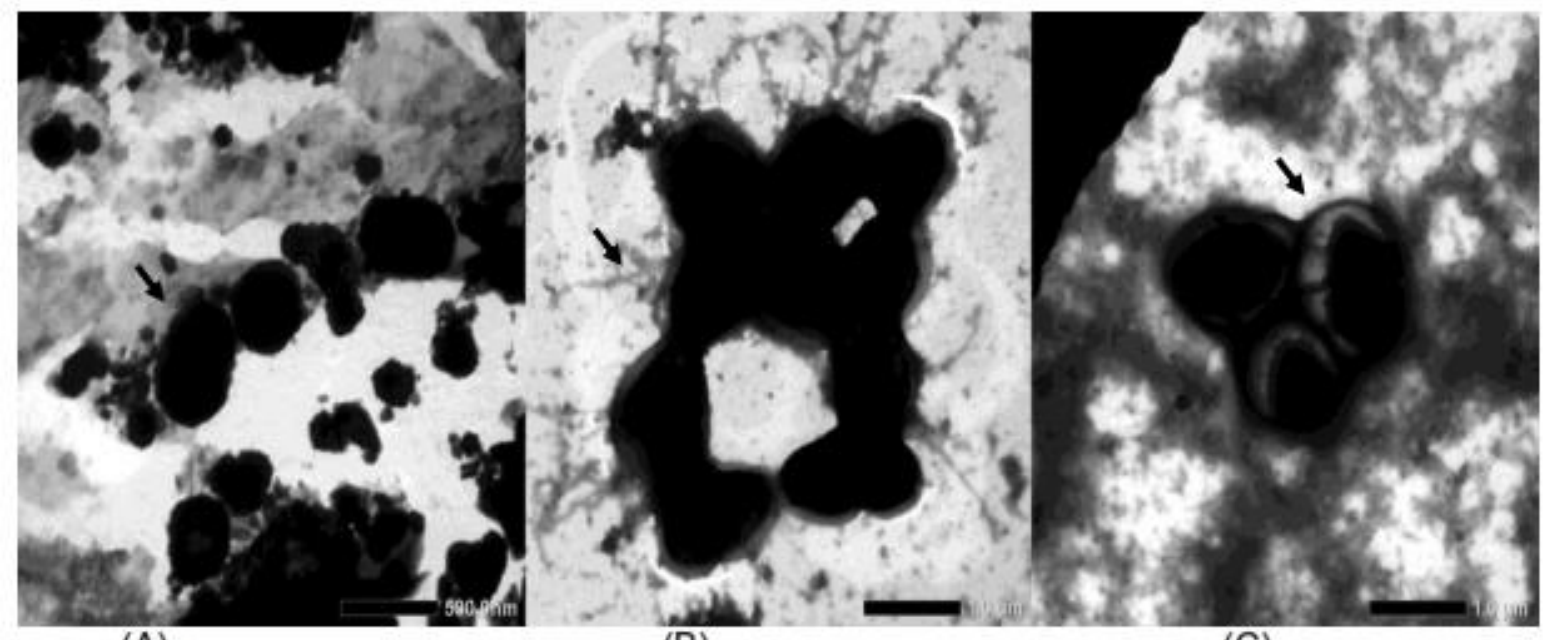

(A)
(B)
(C)

Fig. 2 Biofilm formation in the broth cultures observed with transmission electron microscopy and phosphotungstic acid 2\%. a. J-6 strain b. J-7 strain c. J-8 strain. Arrows showed biofilm structure

J-6 strain were identified as cocci, with thick and extensive biofilm structure outside the cell walls. J-7 strain were shown as streptococci, with thin, thread-like form biofilm. This biofilm form was almost similar with biofilm formation found in Staphylococcus, as well as amyloid structure in E. coli [10]. While J-8 strain were also considered as cocci with thick biofilm surrounding the cell. Thus, it is showed that biofilm 
formation were species-dependent as shown by different phase and forms of biofilm. Further identification is still needed in order to determine chemical composition of biofilm in each strain. Observation using Scanning Electron Microscopy (SEM) is also needed in order to see surface structure of biofilm formation.

\section{Conclusions}

Guava seed fermentation was potential to produce biofilm from indigenous lactic acid bacteria candidates. J-6 and J-8 had ability to form biofilm on glass slides, while J-7 strain was unable to form biofilm. Transmission Electron Microscopy observation showed that all the strains had ability to form biofilm in broth cultures, and it was strain-dependent with different phase and forms of biofilm for each strain.

Acknowledgments This work was financially supported by Indonesian Ministry of Research, Technology and Higher Education for State University Operational Assistance Fund 2016- Research Grants Scheme for Faculty of Biology UGM. The author wishes to thanks to Laboratory of SEM, Faculty of Mathematics and Natural Sciences UGM for TEM analysis.

\section{References}

1. Kokare, C.R., S.Chakraborty, Khopade, A.N., Mahadik K.R.: Biofilm: Importance and applications. Indian J.Biotech, 8, 159-168 (2009).

2. Vu, B., Chen, M., Crawford, R. J., Ivanova. E. P.: Bacterial extracellular polysaccharides involved in biofilm. Molecules, 14, 2535-2554 (2009).

3. Kim, J.Y., Jung, C-K., Kim, D-H., Kim, S-B.: Properties of edible biofilm manufactured from yellowfin tuna (Thunnus albacares) skin gelatin. Korean J.Chem.Eng., 29(6), 786-791 (2012).

4. Seixas, R., Varanda, D., Bexiga, R., Tavares, L., Oliveira, M.: Biofilm-formation by Staphylococcus aureus and Staphylococcus epidermidis isolates from subclinical mastitis in conditions mimicking the udder environment. Pol.J.Vet.Sci., 18(4), 787-792 (2015).

5. Fakhouri, F.M., Palmu, P.S.T., Grosso, C.R.F.: Characterization of composite biofilms of wheat gluten and cellulose acetate phthalate. Braz. J. Chem.Eng., 21(02), 261-264 (2004).

6. Nicanor, A.B., Adriana, A.S., Cristina, A.M., Gloria, D.O.: Guava seed storage protein: Fractination and characterization. LWT, 39, 902-910 (2006).

7. Rocha, K.A.P., Norma, G.V, Aurea, B.N, Leopoldo, G., Hernandez, U.J.P., Alfonso, T.S.: Fortification of white bread with guava seed protein isolate. Pak. J. Nutr., 14(11), 828-833 (2015).

8. Tahmourespour, A., Salehi, R., Kermanshahi, R.K.: Lactobacillus acidophilus-derived biosurfactant effect on gtfb and gtfc expression level in streptococcus mutans biofilm cells. Braz J Microbiol., 42, 330-339 (2011).

9. Chapot-Chartier, M.P., Kulakauskas, S.: Cell wall structure and function in lactic acid bacteria. Microb. Cell Fact.,13(1), S9. (2014).

10. Schwartz, K., Syed, A.K., Stephenson, R.E., Rickard, A.H., Boles, B.R.: Functional amyloids composed of phenol soluble modulins stabilize Staphylococcus Aureus Biofilms. PLoS Pathog 8(6), e1002744 (2012). 